Letter to the Editor

\title{
Paracentral acute middle maculopathy associated with Eisenmenger syndrome
}

Tanya Kowalski MD, ${ }^{1}$ Mali Okada MBBS$^{1}$ and Heather G. Mack PhD FRANZCO 2,3,4

1. Royal Victorian Eye and Ear Hospital, East Melbourne, 3002, Australia

2. Eye Surgery Associates, East Melbourne, 3002, Australia

3 Department of Surgery (Ophthalmology), University of Melbourne, Parkville, 3050, Australia

4 Centre for Eye Research Australia, East Melbourne, 3002, Australia

Correspondence: Tanya Kowalski, 32 Gisborne Street, East Melbourne 3002,

Australia

Email: tanya.kowalski@eyeandear.org.au

Received 3 J uly 2020; accepted 22 August 2020

Funding sources / Financial disclosure: None

Conflict of interest: None

Ethics statement: The Royal Victorian Eye and Ear Hospital Human Research Ethics Committee confirmed ethics approval was not required to report the patient's case; the patient's written approval was obtained to publish this report.

This is the author manuscript accepted for publication and has undergone full peer review but has not been through the copyediting, typesetting, pagination and proofreading process, which may lead to differences between this version and the Version of Record. Please cite this article as doi: 10.1111/ceo.13853

This article is protected by copyright. All rights reserved. 
A 34-year-old male presented with a 2-day history of blurred central vision in the right eye upon waking. He reported a fall the day prior but denied any loss of consciousness or head strike. His medical history was notable for cyanotic Eisenmenger syndrome secondary to a ventricular septal defect (VSD), and obesity, with a body mass index of 30. His VSD was conservatively managed; his medications included warfarin, metoprolol, sildenafil and bosentan. He was a non-smoker, not diabetic, had no known systemic hypertension and was not a migraineur. He reported no excess caffeine consumption. There was no history of recent ophthalmic or other surgery.

At presentation his best corrected visual acuities (BCVA) were right $6 / 12$ and left 6/6. Anterior segments were unremarkable. On fundoscopy, an area of focal retinal oedema and pallor was seen in the right superior macula (Figure 1A). There was associated mild retinal venular dilatation and tortuosity which was also seen in the left eye ( Figure 1B). His optic nerves were normal. Closing retinal venous pressure was not elevated. Intraocular pressures were right $18 \mathrm{mmHg}$ and left $17 \mathrm{mmHg}$. Optical coherence tomography (OCT) of the right eye showed several parafoveal foci of intraretinal hyperreflectivity ( Figure 1C). The left fovea appeared normal (Figure 1D). Blood pressure was $117 / 85 \mathrm{mmHg}$. Haemoglobin was $218 \mathrm{~g} / \mathrm{L}$ (normal 130-180 $\mathrm{g} / \mathrm{L}$ ), haematocrit was $65 \%$ (normal $40-54 \%$ ), and oxygen saturation ranged between $75-88 \%$ at rest (normal 95-100\%). The Royal Victorian Eye and Ear Hospital Human Research Ethics Committee confirmed ethics approval was not required to report the patient's case; the patient's written approval was obtained to publish this report.

Figure 1: At presentation: Colour fundus photographs of the (A) right and (B) left eyes demonstrating right superior macular whitening with bilateral mild retinal venular dilatation and tortuosity. No cilioretinal artery is present. OCT through the (C) right and (D) left maculae demonstrating multiple parafoveal hyperreflective 
bands in the right inner nuclear layer (arrows) and a normal left fovea. There was a single small retinal haemorrhage seen in the right inferior periphery (not shown).

The patient had clinical and OCT findings consistent with right paracentral acute middle maculopathy (PAMM). Unilateral non-ischaemic central retinal vein occlusion was ruled out by the normal central retinal vein closing pressure and bilaterality of retinal venular changes, and hypertensive retinopathy was excluded on the basis of normal blood pressure. Acute macular neuroretinopathy was ruled out by the absence of reddish-brown perifoveal petaloid lesions and the OCT lesion location in the inner nuclear layer.

Eight months after presentation his inferior central scotoma had diminished in size and was only noticeable on monocular vision. His BCVA improved to 6/7.5 in the right eye and although the retinal vascular tortuosity persisted, the right macular lesion was no longer clinically visible. Follow-up fundus photographs, OCT and OCTangiography (OCTA)

demonstrated resolution of the fundus lesion and reduced capillary density (Figure 2).

Figure 2: At month 8: Colour fundus photographs of the (A) right and (B) left eyes demonstrating resolution of right retinal whitening. OCT through the maculae showing (C) right inner nuclear thinning (arrow) and (D) left normal fovea. En face OCTA of (E) right and (F) left maculae showing reduced capillary density in the right eye at the level of the intermediate capillary plexus (arrow), in the area corresponding to previous retinal hyperreflectivity.

Paracentral acute middle maculopathy is a recently described spectral domain OCTbased diagnosis, characterised by hyperreflective lesions in the inner nuclear layer, with a frequently normal fundus examination. ${ }^{1}$ It is thought to reflect a watershed- 
like ischaemia of the middle retinal layers, with OCTA changes reported in the intermediate and deep capillary plexa. Associations with PAMM include a range of retinal vascular conditions such as diabetic retinopathy, retinal vascular occlusion, Purtscher retinopathy, sickle cell disease, hypertensive retinopathy, and global ocular ischaemia. ${ }^{2}$ It has been observed in inflammatory ocular conditions where the mechanism is postulated to be transient occlusion of the retinal capillary plexa and/or vasculitic occlusive retinopathy. ${ }^{1}$ It has also been documented following intraocular and extraocular surgery, where the mechanism has been postulated to be spasm of the central retinal arteriole compounded by periocular anaesthesia, elevated intra-ocular pressure and in some cases pre-existing vasculopathic risk factors including hypotension, dyslipidaemia, and caffeine consumption. ${ }^{1}$ No treatment currently exists. Lesions of PAMM typically progress to atrophy of the inner nuclear layer, and many patients describe a persisting scotoma.

Eisenmenger syndrome is a congenital cardiac defect that causes left-to-right intracardiac shunting, pulmonary hypertension with subsequent right-to-left shunting and chronic systemic hypoxia. Secondary erythrocytosis causes blood hyperviscosity, which can lead to thromboembolic events. Retinal vascular dilatation and tortuosity have been previously recognised and correlated with the extent of arterial oxygen saturation. ${ }^{3}$ Papilloedema, optic disc hypoplasia, microaneurysms, blot haemorrhages, capillary dilation in the peripheral retina and bilateral central retinal vein occlusion have also been described.

Our patient had typical Eisenmenger syndrome with pulmonary hypertension, chronic hypoxia, polycythaemia and retinal vascular dilatation and tortuosity. Given his relatively young age and lack of precipitating and vasculopathic risk factors, we postulate the mechanism of his PAMM was hypoxia, possibly compounded by sildenafil nocturnal hypotension, with reduced perfusion in the intermediate vascular plexus. Unlike the previous report of phosphodiesterase- 5 inhibitor-associated PAMM he did not have a cilioretinal artery. ${ }^{4}$ Micro-emboli are less likely; no neuro-imaging 
is available to test this hypothesis. Hyperviscosity is a less likely mechanism in the absence of retinal vein occlusion. ${ }^{5}$

Advances in the management of complex congenital cardiac disease have improved survival outcomes in patients with associated chronic sequelae including Eisenmenger syndrome. Eyecare providers should be cognisant of the spectrum of retinal manifestations in cyanotic heart disease. This is the first reported case to our knowledge of PAMM in a patient with Eisenmenger syndrome. 


\section{REFERENCES}

1. Moura-Coelho N, Gaspar T, Ferreira JT et al. Paracentral acute middle maculopathy-review of the literature. Graefes Arch Clin Exp Ophthalmol 2020; https://doi.org/10.1007/s00417-020-04826-1

2. Chen X, Rahimy E, Sergott RC, et al. Spectrum of retinal vascular diseases associated with paracentral acute middle maculopathy. Am J Ophthalmol 2015; 160(1):26-34.

3. Mansour AM, Bitar FF, Traboulsi El et al. Ocular pathology in congenital heart disease. Eye 2005; 19(1):29-34.

4. Sebastiani S, Pellegrini M, Giannaccare G, Sarraf D. Paracentral acute middle maculopathy associated with phosphodiesterase- 5 inhibitor therapy. Retin Cases Brief Rep 2018; 10.1097//CB.0000000000000839.

doi:10.1097/ICB.0000000000000839

5. Rodriguez N, Eliott D. Bilateral central retinal vein occlusion in Eisenmenger syndrome. AmJ Ophthalmol 2001; 132(2):268-269. 


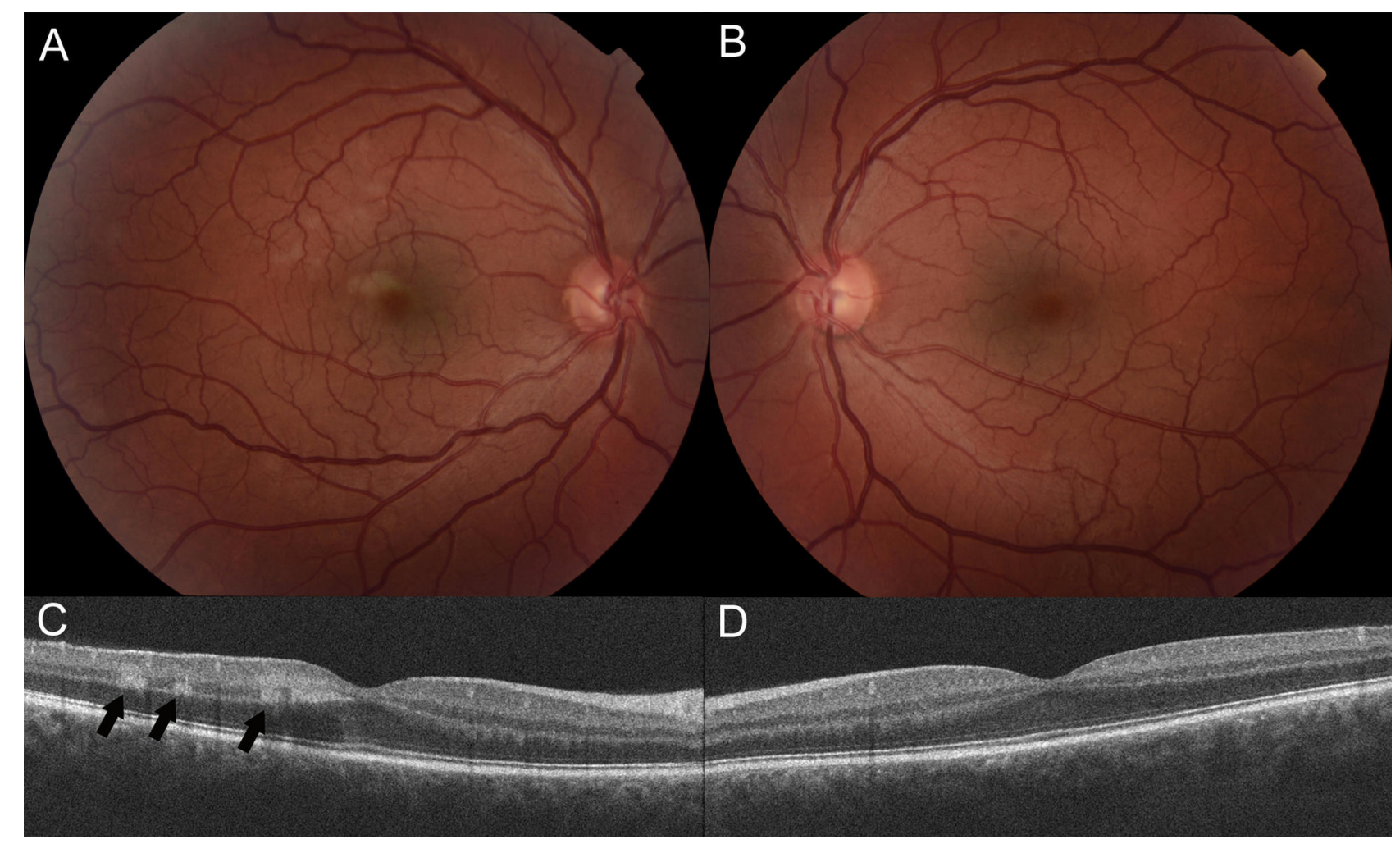

CEO_13853_CEO-20-07-0959 figure 1.tif

This article is protected by copyright. All rights reserved. 


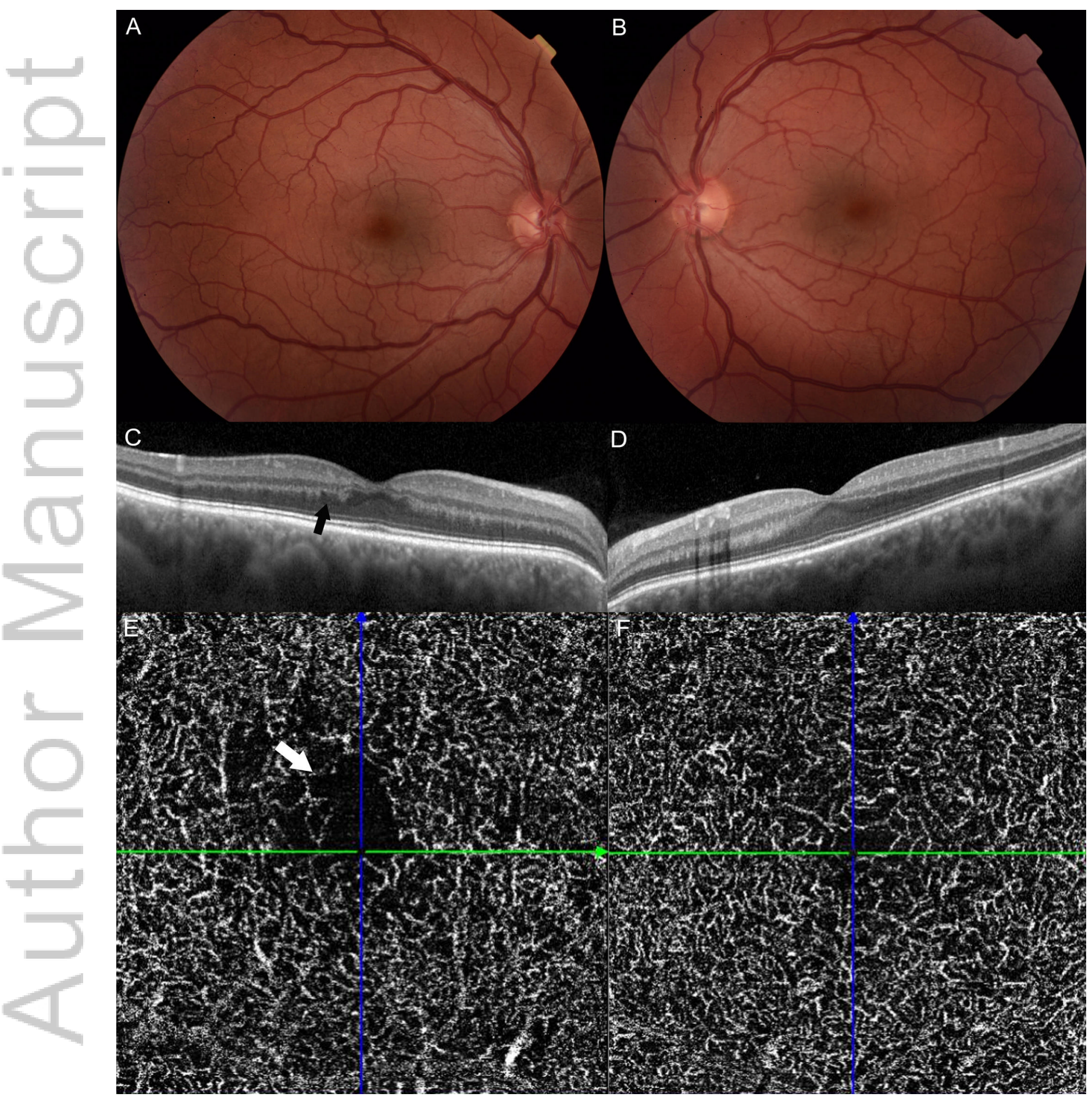

CEO_13853_CEO-20-07-0959 figure 2.tif 


\section{University Library}

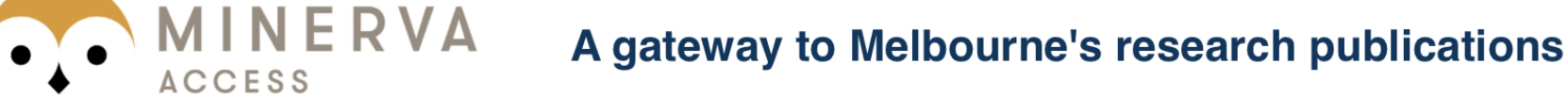

Minerva Access is the Institutional Repository of The University of Melbourne

Author/s:

Kowalski, T;Okada, M;Mack, HG

Title:

Paracentral acute middle maculopathy associated with Eisenmenger syndrome

Date:

2020-09-16

Citation:

Kowalski, T., Okada, M. \& Mack, H. G. (2020). Paracentral acute middle maculopathy associated with Eisenmenger syndrome. CLINICAL AND EXPERIMENTAL

OPHTHALMOLOGY, 48 (8), pp.1106-1108. https://doi.org/10.1111/ceo.13853.

Persistent Link:

http://hdl.handle.net/11343/276308 